\title{
Audio Source Separation With a Single Sensor
}

\author{
Laurent Benaroya, Frédéric Bimbot, and Rémi Gribonval
}

\begin{abstract}
In this paper, we address the problem of audio source separation with one single sensor, using a statistical model of the sources. The approach is based on a learning step from samples of each source separately, during which we train Gaussian scaled mixture models (GSMM). During the separation step, we derive maximum a posteriori (MAP) and/or posterior mean (PM) estimates of the sources, given the observed audio mixture (Bayesian framework). From the experimental point of view, we test and evaluate the method on real audio examples.
\end{abstract} tion.

Index Terms-Audio source separation, Bayesian source separa-

\section{INTRODUCTION}

$\mathbf{S}$ OURCE SEPARATION is an increasingly popular theme in the field of signal processing, especially since new tools, such as independent component analysis (ICA) have been proposed, developed, and improved [2], [5], [7], [13].

ICA has many applications, on biomedical, functional magnetic resonance imaging data for instance, as well as applications in speech processing and audio source separation.

The source separation problem can be formulated as an equation

$$
x_{i}=\sum_{j=1}^{m} a_{i j} s_{j}
$$

where $m$ sources $s_{j}$ with amplitude factors $a_{i j}$ are assumed to be summed to form a collection of $n$ sensor signals $x_{i}$. This case is classically refered to as the linear instantaneous mixture. Note that hypotheses such as independence or non-Gaussianity of the sources usually lead to a solution [11].

Two different cases may be distinguished.

1) The number of sensors $n$ is greater or equal to the number of sources $m$. In this particular case, the estimation of the mixing matrix $A=\left(a_{i j}\right)$ happens to be very useful, as the sources may be recovered via the pseudo-inverse of this matrix.

2) The number of sensors $n$ is less than the number of sources $m$. In this case (known as the under-determined case), the estimation of the matrix $A$ is not sufficient to recover the sources.

Manuscript received March 7, 2003; revised September 21, 2004. The associate editor coordinating the review of this manuscript and approving it for publication was Dr. Futoshi Asano.

The authors are with the IRISA (CNRS \& INRIA), METISS, Campus de Beaulieu, 35042 Rennes Cedex, France (e-mail: lbenaroy@irisa.fr; bimbot@irisa.fr; remi@irisa.fr).

Digital Object Identifier 10.1109/TSA.2005.854110

\section{A. Presentation}

The present article addresses an extreme situation of the second case (under-determined) [13]. We study here the case of a single sensor, with two sources, which is a very specific case, as the mixing equation is reduced to $x=s_{1}+s_{2}$. Here are the main features of this work.

- We use a source model. Building a good model of each source is crucial and it must exploit some knowledge on the sources. In this respect, the approach may not be qualified as "blind" estimation, contrary to classical (even under-determined) cases. In this paper, we address the case of audio sources, of which we build (or assume) statistical models.

- There is a natural formalism for the single sensor case: the Bayesian formalism. This formalism is based on a statistical framework, as the phenomena we observe are variable. It makes it possible to take into account both the additive setting, which yields a likelihood function, and the source models, which provide a priori densities and correspond to prior knowledge on the problem. In practice, we consider a training step in which model parameters of each sources are estimated separately. We then make use of this prior information in the separation step.

Even though we consider, in this study, the special case of two sources with one single sensor, many results can be generalized to more sources (at least theoretically).

\section{B. Formalism}

In a probabilistic formalism, the sources can be estimated through a maximum likelihood (ML) estimate as the mixing equation (1) leads to the definition of a likelihood function

$$
\left(\hat{s}_{1}, \hat{s}_{2}\right)=\arg \max _{s_{1}, s_{2}} p\left(x \mid s_{1}, s_{2}\right)
$$

where $x$ is the observed signal, whereas $s_{i}, i=1,2$ are the sources which are to be estimated. The problem with the ML approach is that there are multiple solutions, since the system is underdetermined.

It is therefore natural to introduce the a posteriori probability distribution for the sources, in a Bayesian formalism

$$
p\left(s_{1}, s_{2} \mid x\right) \propto p\left(x \mid s_{1}, s_{2}\right) p_{1}\left(s_{1}\right) p_{2}\left(s_{2}\right)
$$

where $p\left(x \mid s_{1}, s_{2}\right)$ is the likelihood function and $p_{1}\left(s_{1}\right), p_{2}\left(s_{2}\right)$ correspond to the prior knowledge about the sources. Here the sources are supposed to be independent, i.e., $p\left(s_{1}, s_{2}\right)=p_{1}\left(s_{1}\right) \cdot p_{2}\left(s_{2}\right)$. 
Then, the maximum a posteriori estimator may lead to a solution for the source separation problem

$$
\left.\left(\hat{s}_{1}, \hat{s}_{2}\right)=\arg \max _{s_{1}, s_{2}}\right) p\left(s_{1}, s_{2} \mid x\right) .
$$

Relation (3) is the basis for the estimation of the sources, as it permits to take into account both the additive setting through the likelihood function, and the prior information about the sources via the a priori densities. The parameters of these prior densities (covariance matrices, for instance) are estimated in an off-line training step.

Some previous attempts have been made to solve the source separation problem with one single microphone [4]. In particular, the method proposed in [15] is close to our approach as it uses hidden Markov models and filter theory. Our work provides mathematically grounded algorithms and generalizes the approach to a wide range of statistical models and estimation criteria.

\section{Bayesian Approach}

Several methods in ICA or even in "noisy" principal component analysis (PCA) [5], [16] rely on the Bayesian formalism. In the case of the instantaneous linear mixture of $m$ sources $s$ into $n$ sensors $x$, the basic equation (1) becomes: $x=A s+b$, where $b$ is some white noise (Gaussian distributed for instance).

In this case, the noise distribution corresponds to the likelihood function, because we have: $p(x \mid s, A)=p(x-A s)=$ $p(b)$. In the particular case of Laplacian distributed sources (as prior distributions), the mixing matrix $A$ may be estimated via the maximum a posteriori of the distribution of $s$ conditionally to $x$ (MAP criterion)

$$
\hat{s}=\arg \max _{s} p(s \mid x, A)=\arg \max _{s} p(x \mid s, A) \cdot p(s) .
$$

Generally speaking, when the prior laws are unknown, but the independence of the sources is assumed, the sources may be estimated through a semi-parametric approach [1].

In this study, the models behind the prior densities $p_{1}, p_{2}$ are more specific, though the formalism (i.e., the Bayesian point of view) is the same. In our approach, we use prior information about characteristic Power Spectral Densities (PSD) of each source in order to achieve the source separation. This information may be obtained in a prior training step on separated excerpts of the sources.

\section{Organization of the Paper}

This paper is organized as follows. In Section II, we recall some basics of the Bayesian theory and we describe the classical Wiener filtering approach for stationary sources. In Section III, we make use of the Bayesian formalism in order to derive Wiener estimators in the case of non-Gaussian priors. In Section IV, we present the resulting separation algorithm in the short term Fourier transform (STFT) domain. In Section V, we describe evaluation criteria which we use in the experiments. Finally, in Section VI, we test and evaluate the proposed approach on a real audio excerpt of Jazz music.

\section{WIENER FILTERING}

\section{A. Bayesian Formalism}

1) Framework: As explained in the introduction, the Bayesian formalism offers a natural framework in order to incorporate prior knowledge in an estimation problem. In this section, we recall how this framework can be used for estimating a parameter $\theta$, given observed data $x$.

First, we assume that we are given a parametric statistical model, $f(x \mid \theta)$, where $x$ represents the observed data. $\theta$ is the only unknown parameter (or set of parameters) which belongs to a finite dimensional vector space. The density $f(x \mid \theta)$, from which the data $x$ is drawn, is called the likelihood function as a function of $\theta$.

Then, we define the a priori distribution $\pi(\theta)$ of the parameter $\theta$, which represents the knowledge we have about this parameter, before observing the data $x$. This leads to the definition of the a posteriori density, according to Bayes law

$$
p(\theta \mid x) \propto f(x \mid \theta) \pi(\theta) .
$$

From this distribution, the estimation of parameter $\theta$ is possible and, in a sense, the notion of a posteriori law is a key notion in the Bayesian theory.

2) Estimation and Cost Function: We study now the estimation of the parameter $\theta$, according to the observed data $x$. To do this, we define a cost function $C(\alpha, \theta)$.

This cost $C(\alpha, \theta)$ represents the cost of replacing the true value of the parameter $\theta$ with its estimate $\alpha$.

The estimation of the parameter $\theta$ is done by minimizing the mean cost over all possible values of $\theta$, according to its posterior density

$$
\alpha_{\mathrm{opt}}=\arg \min _{\alpha} \int_{\theta} C(\alpha, \theta) f(x \mid \theta) \pi(\theta) d \theta .
$$

In the case of a quadratic $\operatorname{cost} C(\alpha, \theta)=\|\alpha-\theta\|_{2}^{2}$, the Bayesian estimator is the conditional Posterior Mean (PM): $E(\theta \mid x)$. There exists another standard cost function $C(\alpha, \theta)=1-\delta_{\alpha-\theta}$ ( $\delta$ is the Dirac distribution). In this case, the corresponding Bayesian optimal estimator is the MAP

$$
\alpha_{\mathrm{opt}}=\arg \max _{\theta} f(x \mid \theta) \pi(\theta) .
$$

\section{B. Bayesian Formulation of the Wiener Filter}

Suppose $s_{1}$ and $s_{2}$ are two Gaussian processes, independent, centered, and with covariance matrices $\Sigma_{1}$ and $\Sigma_{2}$. We observe a noisy realization of the sum of the two processes, $x=s_{1}+$ $s_{2}+b$, where $b$ is some Gaussian white noise of variance $\sigma^{2}$.

As presented in the introduction, we have the following likelihood function: $p\left(x \mid s_{1}, s_{2}\right)=p(b)$ and prior density: $p\left(s_{1}, s_{2}\right)=p\left(s_{1}\right) \cdot p\left(s_{2}\right)$. If we further suppose that the noise component is Gaussian distributed, the likelihood function $p\left(x \mid s_{1}, s_{2}\right)$ becomes

$$
p\left(x \mid s_{1}, s_{2}\right)=p(b)=\mathrm{g}\left(x-s_{1}-s_{2}, \sigma^{2} I\right)
$$


where $\mathrm{g}(y, \Sigma)$ is the Gaussian-centered distribution

$$
g(y, \Sigma)=\frac{1}{(2 \pi)^{N / 2}} \frac{1}{\sqrt{|\operatorname{det} \Sigma|}} \exp \left[-\frac{1}{2} y^{T} \Sigma^{-1} y\right]
$$

with $N$ being the dimension of the observation $y$.

Concerning the prior densities, we may assume that $p\left(s_{1}\right)=$ $\mathrm{g}\left(s_{1}, \Sigma_{1}\right), p\left(s_{2}\right)=\mathrm{g}\left(s_{2}, \Sigma_{2}\right)$. In this setting, the likelihood $p\left(x \mid s_{1}, s_{2}\right)$ is the parametric law of the observation $x$, whereas $s_{1}$ and $s_{2}$ are the parameters to be estimated. $p\left(s_{1}\right)$ and $p\left(s_{2}\right)$ are the a priori laws over the parameters, which represents knowledge about these parameters before observing $x$. In this section, we assume a Gaussian a priori law.

Relying on Bayes law, the following expression for the a posteriori law can be derived:

$$
\begin{aligned}
-\log p\left(s_{1}, s_{2} \mid x\right)= & \frac{1}{2 \sigma^{2}}\left\|x-s_{1}-s_{2}\right\|_{2}^{2} \\
& +\frac{1}{2} s_{1}^{T} \Sigma_{1}^{-1} s_{1}+\frac{1}{2} s_{2}^{T} \Sigma_{2}^{-1} s_{2}+\text { cte. }
\end{aligned}
$$

We deduce the MAP estimator for $s_{1}$ and $s_{2}$ from this formula

$$
\begin{aligned}
& \hat{s}_{1}=\Sigma_{1}\left[\Sigma_{1}+\Sigma_{2}+\sigma^{2} I\right]^{-1} x \\
& \hat{s}_{2}=\Sigma_{2}\left[\Sigma_{1}+\Sigma_{2}+\sigma^{2} I\right]^{-1} x .
\end{aligned}
$$

In the case of a "vanishing" noise, i.e., $\sigma \rightarrow 0$, the estimator converges toward the Wiener estimator.

From expression (4), we see that the posterior distribution $p\left(s_{1}, s_{2} \mid x\right)$ is a Gaussian distribution, as the expression inside the brackets is a quadratic form in $s_{1}$ and $s_{2}$. We conclude that the MAP and PM estimators are, in that case, identical.

\section{Stationary Processes}

In the specific case when $s_{1}$ and $s_{2}$ are stationary and (approximately) circular processes (i.e., with a Toeplitz covariance matrix) $s_{1}$ and $s_{2}$, the basis $\mathcal{B}$ which makes both covariance matrices diagonal is the discrete Fourier basis, which vectors are $\{\mathcal{F} f[n]=(1 / \sqrt{N}) \exp (2 \pi i f n / N)\}_{0 \leq f<N}$, where $\mathcal{F}$ denotes the discrete Fourier transform operator and $f$ denotes the frequency index.

In this case, the Wiener filtering can be interpreted as the following operation in the frequency domain

$$
\begin{aligned}
& \hat{\mathcal{F}} s_{1}[f]=\frac{\sigma_{1}^{2}[f]}{\sigma_{1}^{2}[f]+\sigma_{2}^{2}[f]} \cdot \mathcal{F} x[f] \\
& \hat{\mathcal{F}}_{s_{2}[f]}=\frac{\sigma_{2}^{2}[f]}{\sigma_{1}^{2}[f]+\sigma_{2}^{2}[f]} \cdot \mathcal{F} x[f] .
\end{aligned}
$$

\section{Limits and Extensions}

Let us recall the set of hypotheses made so far.

- The a priori knowledge concerning the sources is reduced to the knowledge of the covariance matrices, which corresponds to Power Spectral Densities (PSD), in the stationary case.

- The stochastic processes $s_{1}$ and $s_{2}$ are assumed to be Gaussian; equivalently we restrict the problem to linear estimators.

- Both processes $s_{1}$ and $s_{2}$ are stationary and circular.
As audio signals are generally non-Gaussian and nonstationary, the previous method may not be applied directly. The approach must be generalized to other prior densities, through the Bayesian framework.

This suggests to extend classical Wiener filtering to different kind of prior densities, in particular to non-Gaussian unimodal densities, to Gaussian mixture models and even to more complex models.

\section{EXTENSIONS OF WIENER FILTERS TO NON-GAUSSIAN PRIORS}

\section{A. Non-Gaussian Unimodal Densities}

The Wiener filter approach can be extended to other families of unimodal densities, for instance generalized Gaussian densities

$$
\mathrm{G}(y, \alpha, \Sigma) \propto \exp \left[-\beta(\alpha)\left\|\Sigma^{-1 / 2} y\right\|_{\alpha}^{\alpha}\right]
$$

where $\|y\|_{\alpha}^{\alpha}=\Sigma_{k}|y[k]|^{\alpha}$. We recall that $\beta(\alpha)=$ $\left[(\Gamma(3 / \alpha)) /(\Gamma(1 / \alpha)]^{\alpha / 2}\right.$

The Bayesian model now takes the following form:

$$
\begin{aligned}
& p\left(x \mid s_{1}, s_{2}\right)=\mathrm{g}\left(x-s_{1}-s_{2}, \sigma^{2} I\right) \quad \text { likelihood function } \\
& \left.\begin{array}{l}
p\left(s_{1}\right)=\mathrm{G}\left(s_{1}, \alpha_{1}, \Sigma_{1}\right) \\
p\left(s_{2}\right)=\mathrm{G}\left(s_{2}, \alpha_{2}, \Sigma_{2}\right)
\end{array}\right\} \quad \text { prior densities. }
\end{aligned}
$$

The a priori law of the sources $s_{1}$ and $s_{2}$ are thus generalized Gaussian densities.

Using Bayes law, the a posteriori law becomes

$$
\begin{aligned}
& -\log p\left(s_{1}, s_{2} \mid x\right)=\frac{1}{2 \sigma^{2}}\left\|x-s_{1}-s_{2}\right\|_{2}^{2} \\
& \quad+\beta\left(\alpha_{1}\right)\left\|\Sigma_{1}^{-1 / 2} s_{1}\right\|_{\alpha_{1}}^{\alpha_{1}}+\beta\left(\alpha_{2}\right)\left\|\Sigma_{2}^{-1 / 2} s_{2}\right\|_{\alpha_{2}}^{\alpha_{2}}+\text { cte. }
\end{aligned}
$$

It is sometimes possible to find an expression (in some cases, an analytic one) for the MAP and PM estimators of $s_{1}$ and $s_{2}$. Let us have a look at the MAP estimator in some particular cases.

\section{1) Particular Cases:}

Both Sources Have Laplacian Prior Densities: In the case $\alpha_{1}=\alpha_{2}=1$, i.e., both prior densities are Laplacian laws and the covariance matrices are diagonal, we obtain the following MAP estimators, in the noiseless case

$$
\begin{aligned}
& \hat{s}_{1}[k]= \begin{cases}x[k], & \text { if } \sigma_{1}[k]>\sigma_{2}[k] \\
0, & \text { otherwise }\end{cases} \\
& \hat{s}_{2}[k]= \begin{cases}x[k], & \text { if } \sigma_{2}[k]>\sigma_{1}[k] \\
0, & \text { otherwise. }\end{cases}
\end{aligned}
$$

One Laplace Source and One Gaussian Source: Assuming now that source $s_{1}$ has a laplacian prior density, i.e., $\alpha_{1}=1$, with diagonal covariance matrix, whereas $s_{2}$ is a Gaussian white noise of variance $\sigma_{2}^{2}$, that is $\alpha_{2}=2$.

Then the MAP estimator for $s_{1}$ is the coefficients shrinkage proposed by Donoho in [8]

$$
\hat{s}_{1}[k]=\operatorname{sign}(x[k]) \cdot \max [|x[k]|-\lambda[k], 0]
$$

where $\lambda[k]=\left(\sqrt{2} \sigma_{2}^{2}\right) /\left(\sigma_{1}[k]\right)$. 
In this case, the second source may be considered as a noise and the expression (5) may be interpreted as a reduction of the corrupted observed signal $x$ from a quantity proportional to the noise variance. If the sources are expressed in a wavelet basis, this is often refered to as wavelet-shrinkage and this is a powerful tool for denoising purposes.

More General Case: $\alpha_{1}=\alpha_{2}=\alpha$ In this case (same generalized Gaussian density for each source), it is possible to define a function $h(r ; \alpha)$ if both covariance matrix are diagonal. $h(r ; \alpha)$ is the function

$$
h(r ; \alpha)=\frac{r^{\left(\frac{\alpha}{\alpha-1}\right)}}{1+r^{\left(\frac{\alpha}{\alpha-1}\right)}}
$$

in the case $\alpha>1$. We obtain (noiseless case)

$$
\begin{aligned}
& \hat{s}_{1}[k]=h\left(\frac{\sigma_{1}[k]}{\sigma_{2}[k]} ; \alpha\right) x[k] \\
& \hat{s}_{2}[k]=\left[1-h\left(\frac{\sigma_{1}[k]}{\sigma_{2}[k]} ; \alpha\right)\right] x[k] .
\end{aligned}
$$

This is just a generalization of the Wiener filter formula with a different shape for the weighting function $h$.

\section{B. Case of Gaussian Mixture Models}

The above developments can be viewed as examples of what can be done using the Bayesian framework, in the case of unimodal densities.

For dealing with nonstationary signals, it is necessary to consider other families of models for the sources. In this section, we study the case of Gaussian Mixture prior densities (GMM priors) [6], in line with former work in the field of speech processing, where parent approaches have been used to enhance the robustness of speech recognition in noisy environments (see for instance [17]-[19])

$$
\mathcal{G}\left(y,\left\{\varpi^{(i)}\right\},\left\{\Sigma^{(i)}\right\}\right)=\sum_{i=1}^{K} \varpi^{(i)} \mathrm{g}\left(y, \Sigma^{(i)}\right)
$$

where $g$ is the Gaussian function and $\sum_{i=1}^{K} \varpi^{(i)}=1$.

As a generative model, the Gaussian mixture model assumes that an observation is obtained by first selecting one active component within the $K$ Gaussians in the mixture (following the probability distribution $\left\{\varpi^{(i)}\right\}$ ) and then generating a Gaussian observation following $\mathrm{g}\left(y, \Sigma^{(i)}\right)$ for the active component.

For source separation, the Gaussian mixture model permits to deal with multiple covariance matrices, that is multiple power spectral densities (PSD) shapes, in the case of frequency domain filtering.

In the Bayesian formalism, we obtain the following prior densities:

$$
\begin{aligned}
& p\left(s_{1}\right)=\sum_{i=1}^{K_{1}} \varpi_{1}^{(i)} \frac{\exp \left[-\frac{1}{2} s_{1}^{T} \Sigma_{1}^{(i)^{-1}} s_{1}\right]}{(2 \pi)^{N / 2}\left|\operatorname{det}\left(\Sigma_{1}^{(i)}\right)\right|^{1 / 2}} \\
& p\left(s_{2}\right)=\sum_{j=1}^{K_{2}} \varpi_{2}^{(j)} \frac{\exp \left[-\frac{1}{2} s_{2}^{T} \Sigma_{2}^{(j)^{-1}} s_{2}\right]}{(2 \pi)^{N / 2}\left|\operatorname{det}\left(\Sigma_{2}^{(j)}\right)\right|^{1 / 2}}
\end{aligned}
$$

with $\Sigma_{i=1}^{K_{1}} \varpi_{1}^{(i)}=\Sigma_{j=1}^{K_{2}} \varpi_{2}^{(j)}=1$.
Here, the MAP estimation is not tractable directly. In order to get back to the Gaussian case (which is solved with Wiener filters), we introduce hidden variables $q_{1}$ and $q_{2}$ which are associated with the active components in both GMM models, i.e., the Gaussian densities from which the sources data were most likely generated. This is a typical incomplete data setting.

In other words, the following likelihood and prior densities for the hidden $q_{i}$ process $(i=1,2)$ are considered

$$
\begin{aligned}
p\left(s_{i} \mid q_{i}=k\right) & =\frac{\exp \left[-\frac{1}{2} s_{i}^{T} \Sigma_{i}^{(k)^{-1}} s_{i}\right]}{(2 \pi)^{N / 2}\left|\operatorname{det}\left(\Sigma_{i}^{(k)}\right)\right|^{1 / 2}} \\
p\left(q_{i}=k\right) & =\varpi_{i}^{(k)} .
\end{aligned}
$$

The estimators are thus calculated conditionally to the hidden state couple $\left(q_{1}, q_{2}\right)$.

1) First Step: State Estimation: As the couple of states $\left(q_{1}, q_{2}\right)$ is generally unknown, we have to estimate this couple.

If the states are $q_{1}=i$ and $q_{2}=j$ (that is to say, if we know the active components in both mixture models), then $s_{1}$ has a Gaussian distribution conditionally to $q_{1}$, of covariance matrix $\Sigma_{1}^{(i)}$ and $s_{2}$ has also a Gaussian distribution conditionally to $q_{2}$ with covariance matrix $\Sigma_{2}^{(j)}$. We deduce that the sum $x=$ $s_{1}+s_{2}+b$ has Gaussian distribution conditionally to $\left(q_{1}, q_{2}\right)$ with covariance matrix $\Sigma_{1}^{(i)}+\Sigma_{2}^{(j)}+\sigma^{2} I$.

We deduce then the following posterior formula:

$$
\begin{aligned}
p(i, j \mid x) & \propto p(x \mid i, j) \cdot p(i) \cdot p(j) \\
& \propto \varpi_{1}^{(i)} \varpi_{2}^{(j)} \operatorname{gauss}\left(x, \Sigma_{1}^{(i)}+\Sigma_{2}^{(j)}+\sigma^{2} I\right) .
\end{aligned}
$$

This is the a posteriori law for the couple of components $(i, j)$ for both mixture models, conditionally to the observed process $x$. We will note in the following $\gamma_{i, j}(x)=p(i, j \mid x)$, which is the a posteriori probability that the components $(i, j)$ are active in each respective GMM, when observing $x$.

2) Second Step: Construction of the Filters: If the active states $q_{1}$ and $q_{2}$ are known, then the problem can be solved by the Wiener filter approach, conditionally to the couple $\left(q_{1}, q_{2}\right)$, as both priors are conditionally Gaussian.

We have

$$
\begin{aligned}
-2 \log p\left(s_{1}, s_{2} \mid x, i, j\right) & =\frac{1}{\sigma_{2}}\left\|x-s_{1}-s_{2}\right\|_{2}^{2} \\
+ & s_{1}^{T}\left[\Sigma_{1}^{(i)}\right]^{-1} s_{1}+s_{2}^{T}\left[\Sigma_{2}^{(j)}\right]^{-1} s_{2}+\text { cte. }
\end{aligned}
$$

If $q_{1}=i$ and $q_{2}=j$ are known, we have the conditional Bayesian (Wiener) estimator (as we have seen previously, the conditional MAP and PM estimators coincide)

$$
\begin{aligned}
& E\left(s_{1} \mid i, j\right)=\Sigma_{1}^{(i)}\left[\Sigma_{1}^{(i)}+\Sigma_{2}^{(j)}+\sigma^{2} I\right]^{-1} x \\
& E\left(s_{2} \mid i, j\right)=\Sigma_{2}^{(j)}\left[\Sigma_{1}^{(i)}+\Sigma_{2}^{(j)}+\sigma^{2} I\right]^{-1} x .
\end{aligned}
$$

Maximum a posteriori Estimation: When the active components $q_{1}=i, q_{2}=j$ are not known, they can be estimated as the MAP estimation of $\gamma_{i, j}(x)$ yielding one active component per GMM source model. In that case, we fall back on the Wiener filter setting, using the estimated couple of states. The approach can be understood as an adaptive Wiener filtering process. 
Posterior Mean Estimator: We may also estimate the sources $s_{1}$ and $s_{2}$ through the PM estimator [9].

As we have from Bayes law

$$
\begin{aligned}
p\left(s_{1} \mid x\right) & \propto \sum_{i, j} p\left(s_{1} \mid x, i, j\right) p\left(q_{1}=i, q_{2}=j \mid x\right) \\
& \propto \sum_{i, j} p\left(s_{1} \mid x, i, j\right) \gamma_{i, j}(x) .
\end{aligned}
$$

We deduce the following PM estimator:

$$
\begin{aligned}
E\left(s_{1} \mid x\right) & =\int_{s_{1}} s_{1} p\left(s_{1} \mid x\right) d s_{1} \\
& =\sum_{i, j} \gamma_{i, j}(x)\left[\int_{s_{1}} s_{1} p\left(s_{1} \mid x, i, j\right) d s_{1}\right] \\
& =\sum_{i, j} \gamma_{i, j}(x) E\left(s_{1} \mid x, i, j\right) .
\end{aligned}
$$

Finally

$$
E\left(s_{1} \mid x\right)=\sum_{i=1}^{K_{1}} \sum_{j=1}^{K_{2}} \gamma_{i, j}(x) \cdot \Sigma_{1}^{(i)}\left[\Sigma_{1}^{(i)}+\Sigma_{2}^{(j)}+\sigma^{2} I\right]^{-1} \cdot x
$$

and similarily for $s_{2}$.

Moreover, relying on the above developments

$$
\gamma_{i, j}(x) \propto \varpi_{1}^{(i)} \varpi_{2}^{(j)} \mathrm{g}\left(x, \Sigma_{1}^{(i)}+\Sigma_{2}^{(j)}+\sigma^{2} I\right)
$$

with $\Sigma_{i, j} \gamma_{i, j}(x)=1$.

Thus, the first step consists in computing the posterior probabilities $\gamma_{i, j}(x)$, followed by the computation of weighted Wiener filters. The second step consists in filtering the sources with this adapted filter, with weight coefficients $\gamma_{i, j}(x)$ which thus depend on the observed process $x$.

3) HMM Models: It must be noted that the generalized Wiener filter with GMM models can be extended to HMM models (Hidden Markov Models). Indeed, the only difference is that the weighting probabilities $\gamma_{i, j}(x)$ must then be computed through a forward-backward algorithm, which may result in a greater algorithmic cost ${ }^{1}$.

4) Limitations of the GMM Model: In the context of audio processing, we may observe the same sound corresponding to a similar PSD shape, repeated at different amplitudes and time indexes. If the GMM models are used as described above, there has to be as many Gaussian components as there are different possible amplitudes, although they correspond to the same sound. This is quite restrictive.

This is why we have considered a more elaborate model: the Gaussian scaled mixture model (GSMM), in order to separate

\footnotetext{
${ }^{1}$ The algorithmic complexity of the algorithm with GMM models (which can be viewed as HMM models of order 0$)$ is of order $O\left(Q_{1} \cdot Q_{2}\right)$, where $Q_{1}$ and $Q_{2}$ are the number of Gaussian components in each source model. With fully-connected HMM models of order p, the complexity becomes $O\left(Q_{1}^{p+1}\right.$. $\left.Q_{2}^{p+1}\right)$. As a result, the algorithmic complexity with HMM models may be very high and even untractable in the case of HMM models of order greater than one, unless they are only sparsely connected.
}

the variance shape (PSD), and the amplitude information (gain factor).

\section{Gaussian Scaled Mixture Models}

The GSMM is a mixture of Gaussian scaled densities [14].

A Gaussian scaled density corresponds to a random variable of the form $g_{a}=\sqrt{a} \cdot g$, where $g$ is a Gaussian distributed vector variable with variance $\sigma^{2}$ and $a$ is a nonnegative scalar random variable, which may be drawn according to a prior density $p_{0}(a)$.

Thus the density of the Gaussian scaled variable $y$ is

$$
g_{a}(y \mid a)=\frac{1}{\sqrt{2 \pi a} \sigma} \exp \left[-1 / 2 \frac{\|y\|_{2}^{2}}{a \sigma^{2}}\right] .
$$

The marginal law is

$$
g_{a}(y) \propto \int_{a} \frac{1}{\sqrt{2 \pi a} \sigma} \exp \left[-1 / 2 \frac{\|y\|_{2}^{2}}{a \sigma^{2}}\right] p_{0}(a) d a .
$$

A Gaussian scaled mixture model takes therefore the following form:

$$
\begin{aligned}
& p\left(s_{1} \mid a_{1}^{1}, \ldots, a_{K_{1}}^{1}\right)=\sum_{i=1}^{K_{1}} \varpi_{1}^{(i)} \frac{\exp \left[-\frac{1}{2} s_{1}^{T}\left(a_{i}^{1} \Sigma_{1}^{(i)}\right)^{-1} s_{1}\right]}{\left(2 \pi a_{i}^{1}\right)^{N / 2}\left|\operatorname{det}\left(\Sigma_{1}^{(i)}\right)\right|^{1 / 2}} \\
& p\left(s_{2} \mid a_{1}^{2}, \ldots, a_{K_{2}}^{2}\right)=\sum_{j=1}^{K_{2}} \varpi_{2}^{(j)} \frac{\exp \left[-\frac{1}{2} s_{2}^{T}\left(a_{j}^{2} \Sigma_{2}^{(j)}\right)^{-1} s_{2}\right]}{\left(2 \pi a_{j}^{2}\right)^{N / 2}\left|\operatorname{det}\left(\Sigma_{2}^{(j)}\right)\right|^{1 / 2}}
\end{aligned}
$$

Conditionally to $q_{1}=i, q_{2}=j, a_{i}^{1}$, and $a_{j}^{2}$, the Bayesian estimator (MAP or PM) is (cf. Wiener filter):

$$
\begin{aligned}
& E\left(s_{1} \mid x, i, j, a_{i}^{1}, a_{j}^{2}\right)=a_{i}^{1} \Sigma_{1}^{(i)}\left[a_{i}^{1} \Sigma_{1}^{(i)}+a_{j}^{2} \Sigma_{2}^{(j)}+\sigma^{2} I\right]^{-1} x \\
& E\left(s_{2} \mid x, i, j, a_{i}^{1}, a_{j}^{2}\right)=a_{i}^{2} \sum_{j}^{(i)}\left[a_{i}^{1} \Sigma_{1}^{(i)}+a_{j}^{2} \Sigma_{2}^{(j)}+\sigma^{2} I\right]^{-1} x
\end{aligned}
$$

Conditionally to $a_{1}^{1}, \ldots, a_{K_{1}}^{1}$ and $a_{1}^{2}, \ldots, a_{K_{2}}^{2}$, the weighting probabilities are:

$$
\gamma_{i, j, a_{i}^{1}, a_{j}^{2}}(x) \propto \varpi_{1}^{(i)} \varpi_{2}^{(j)} \operatorname{gauss}\left(x, a_{i}^{1} \Sigma_{1}^{(i)}+a_{j}^{2} \Sigma_{2}^{(j)}+\sigma^{2} I\right)
$$

as $a_{i}^{1} \sum_{1}^{(i)}+a_{j}^{2} \sum_{2}^{(j)}+\sigma^{2} I$ is the covariance matrix of the observed process, conditionally to the couple of states and the amplitudes.

For the posterior mean Bayesian estimator, we should integrate these estimates over all possible values of the amplitude parameters, that is:

$$
\begin{aligned}
E\left(s_{1} \mid x\right)=\sum_{i=1}^{K_{1}} & \sum_{j=1}^{K_{2}} \int_{a_{i}^{1}} \int_{a_{j}^{2}} \gamma_{i, j, a_{i}^{1}, a_{j}^{2}}(x) \\
& \times E\left(s_{1} \mid x, i, j, a_{i}^{1}, a_{j}^{2}\right) p_{0}\left(a_{i}^{1}\right) p_{0}\left(a_{j}^{2}\right) d a_{i}^{1} d a_{j}^{2} .
\end{aligned}
$$




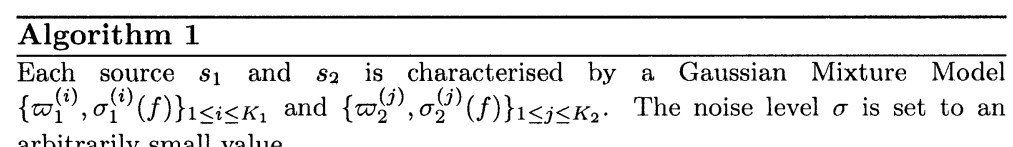
$\left\{\varpi_{1}^{(i)}, \sigma_{1}^{(i)}(f)\right\}_{1 \leq i \leq K_{1}}$ and $\left\{\varpi_{2}^{(j)}, \sigma_{2}^{(j)}(f)\right\}_{1 \leq j \leq K_{2}}$. The noise level $\sigma$ is set to an arbitrarily small value.

1. For each frame index $t$, compute the weighting probabilities :

$$
\begin{gathered}
\gamma_{i, j}(t) \propto \varpi_{1}^{(i)} \varpi_{2}^{(j)} \times \\
\prod_{f} \operatorname{gauss}\left(|\mathcal{S} x(t, f)|, \sigma_{1}^{(i)}(f)^{2}+\sigma_{2}^{(j)}(f)^{2}+\sigma^{2}\right),
\end{gathered}
$$

with $\sum_{i, j} \gamma_{i, j}(t)=1$.

2. Then compute the posterior mean estimator :

$$
\begin{aligned}
& \widehat{\mathcal{S} s_{1}}(t, f)=\sum_{i=1}^{K_{1}} \sum_{j=1}^{K_{2}} \gamma_{i, j}(t) \frac{\sigma_{1}^{(i)}(f)^{2}}{\sigma_{1}^{(i)}(f)^{2}+\sigma_{2}^{(j)}(f)^{2}+\sigma^{2}} \mathcal{S} x(t, f), \\
& \widehat{\mathcal{S}_{2}}(t, f)=\sum_{i=1}^{K_{1}} \sum_{j=1}^{K_{2}} \gamma_{i, j}(t) \frac{\sigma_{2}^{(j)}(f)^{2}}{\sigma_{1}^{(i)}(f)^{2}+\sigma_{2}^{(j)}(f)^{2}+\sigma^{2}} \mathcal{S} x(t, f) .
\end{aligned}
$$

As the integrals may be untractable, we use a maximum likelihood estimation to determine the coefficients $a_{i}^{1}$ and $a_{j}^{2}$, under a positivity constraint and we set the amplitude coefficients to this value instead of integrating out.

We use the following estimation formula:

$$
\left(\widehat{a_{i}^{1}}, \widehat{a_{j}^{2}}\right)=\arg \max _{a_{1} \geq 0, a_{2} \geq 0} \gamma_{i, j, a_{i}^{1}, a_{j}^{2}}(x)
$$

which can be seen as a reweighted positive least square estimate.

\section{SEPARATION AlgORITHM}

As we aim to separate audio sources, which are locally stationary in general, it is natural to work with the short-term Fourier transform (STFT) denoted by $\mathcal{S}$. As this transform is linear, the additive setting remains: $\mathcal{S} x(t, f)=\mathcal{S} s_{1}(t, f)+\mathcal{S} s_{2}(t, f)+\mathcal{S} b(t, f)$. The covariance matrices $\sum_{1}^{(i)}$ and $\sum_{2}^{(j)}$ are assumed to be diagonal, with running element $\sigma_{1}^{(i)}(f)^{2}$ and $\sigma_{2}^{(j)}(f)^{2}$ respectively.

\section{A. GMM Models}

We note $\gamma_{i, j}(x)=\gamma_{i, j}(t)$, the weighting probabilities corresponding to the observed frame $\mathcal{S} x(t, f)$ at time index $t$. The separation algorithm with the GMM models is given in the Algorithm 1, shown at the top of the page.

\section{B. GSMM Models}

In the STFT setting, conditionally to the pair of states $(i, j)$ and to the amplitude parameters $a_{i}^{1}, a_{j}^{2}$, the sources are Gaussian centered processes. Therefore, the observed mixture is also a Gaussian centered process, of diagonal covariance $a_{i}^{1} \sigma_{1}^{(i)}(f)^{2}+$ $a_{j}^{2} \sigma_{2}^{(j)}(f)^{2}+\sigma^{2}$. Then, we have the following likelihood function:

$$
\begin{aligned}
& p\left(x \mid i, j, a_{i}^{1}, a_{j}^{2}\right) \approx \\
& \frac{\exp \left[-\sum_{f} \frac{|\mathcal{S} x(t, f)|^{2}}{2\left(a_{i}^{1} \sigma_{1}^{(i)}(f)^{2}+a_{j}^{2} \sigma_{2}^{(j)}(f)^{2}+\sigma^{2}\right)}\right]}{\prod_{f} \sqrt{2 \pi\left(a_{i}^{1} \sigma_{1}^{(i)}(f)^{2}+a_{j}^{2} \sigma_{2}^{(j)}(f)^{2}+\sigma^{2}\right)}} .
\end{aligned}
$$

The amplitude coefficients can be computed in a Maximum Likelihood scheme, under positivity constraints. It can be shown [3] that (7) can be solved by finding $\left(a_{i}^{1}, a_{j}^{2}\right)$ so as to solve the following system:

$$
\begin{aligned}
& \sum_{f} a_{i}^{1} \sigma_{1}^{(i)}(f)^{2} \\
& \times \frac{|\mathcal{S} x(t, f)|^{2}-\left(a_{i}^{1} \sigma_{1}^{(i)}(f)^{2}+a_{j}^{2} \sigma_{2}^{(j)}(f)^{2}+\sigma^{2}\right)}{\left(a_{i}^{1} \sigma_{1}^{(i)}(f)^{2}+a_{j}^{2} \sigma_{2}^{(j)}(f)^{2}+\sigma^{2}\right)^{2}}=0 \\
& \sum_{f} a_{j}^{2} \sigma_{2}^{(j)}(f)^{2} \\
& \quad \times \frac{|\mathcal{S} x(t, f)|^{2}-\left(a_{i}^{1} \sigma_{1}^{(i)}(f)^{2}+a_{j}^{2} \sigma_{2}^{(j)}(f)^{2}+\sigma^{2}\right)}{\left(a_{i}^{1} \sigma_{1}^{(i)}(f)^{2}+a_{j}^{2} \sigma_{2}^{(j)}(f)^{2}+\sigma^{2}\right)^{2}}=0 .
\end{aligned}
$$

These equations are obtained by differentiating the logarithm of (8) with respect to the amplitude parameters, and introducing Lagrange multipliers in order to incorporate the positivity constraints. They can be solved through an iterative procedure, where the denominator is kept constant [12], leading to the first step as described in Algorithm 2, shown at the top of the next page.

The estimation of the amplitude parameters $a_{1}$ and $a_{2}$ can be interpreted as a match of the squared spectral module of the STFT process $\mathcal{S} x(t, f)$ with the estimated variances $a_{i}^{1} \sigma_{1}^{(i)}(f)^{2}+a_{j}^{2} \sigma_{2}^{(j)}(f)^{2}+\sigma^{2}$, under positivity constrains.

The separation algorithm with the GSMM models is summarized in the Algorithm 2.

\section{EVALUATION CRITERIA}

For the evaluation of the separation experiments, we need to define some criteria, in order to compare the performance of GMM models in various settings (different numbers of components for the model of each source). We suppose that the two original sources $s_{1}$ and $s_{2}$ are uncorrelated and we denote their estimates $\hat{s}_{1}$ and $\hat{s}_{2}$. 


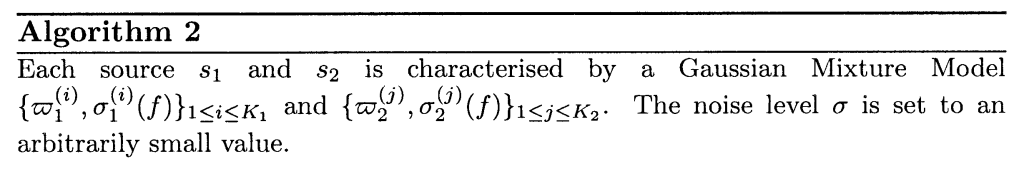

1. For each frame index $t$, compute the amplitude coefficients, by the following iterative procedure :

(a) Initialize $\left[a_{i}^{1}(t)\right]_{0}$ and $\left[a_{j}^{2}(t)\right]_{0}$

(b) At each iteration $\ell$

- evaluate $[r(t, f)]_{\ell}^{2}=$ $\left[a_{i}^{1}(t)\right]_{\ell-1} \sigma_{1}^{(i)}(f)^{2}+\left[a_{j}^{2}(t)\right]_{\ell-1} \sigma_{2}^{(j)}(f)^{2}+\sigma^{2}$

- update $\left[a_{i}^{1}(t)\right]_{\ell}=\left[a_{i}^{1}(t)\right]_{\ell-1} \times$

$\sum_{f} \frac{\sigma_{1}^{(i)}(f)^{2}}{[r(t, f)]_{\ell}^{2}} \frac{|\mathcal{S} x(t, f)|^{2}}{[r(t, f)]_{\ell}^{2}} / \sum_{f} \frac{\sigma_{1}^{(i)}(f)^{2}}{[r(t, f)]_{\ell}^{2}}$

- update $\left[a_{j}^{2}(t)\right]_{\ell}=\left[a_{j}^{2}(t)\right]_{\ell-1} \times$

$\sum_{f} \frac{\sigma_{2}^{(j)}(f)^{2}}{[r(t, f)]_{\ell}^{2}} \frac{|\mathcal{S} x(t, f)|^{2}}{[r(t, f)]_{\ell}^{2}} / \sum_{f} \frac{\sigma_{2}^{(j)}(f)^{2}}{[r(t, f)]_{\ell}^{2}}$

- if convergence then $a_{i}^{1}(t)=\left[a_{i}^{1}(t)\right]_{\ell}$ and $a_{j}^{2}(t)=\left[a_{j}^{2}(t)\right]_{\ell}$ else iterate $(\ell \leftarrow \ell+1)$

2. Compute the weighting probabilities $\gamma_{i, j}(t)$ :

$$
\begin{gathered}
\gamma_{i, j}(t) \propto \varpi_{1}^{(i)} \varpi_{2}^{(j)} \times \\
\prod_{f} \operatorname{gauss}\left(|\mathcal{S} x(t, f)|, a_{i}^{1} \sigma_{1}^{(i)}(f)^{2}+a_{j}^{2} \sigma_{2}^{(j)}(f)^{2}+\sigma^{2}\right),
\end{gathered}
$$

with $\sum_{i, j} \gamma_{i, j}(t)=1$.

3. Finally, filter the observed frame :

$$
\begin{aligned}
& \widehat{\mathcal{S} s_{1}}(t, f)=\sum_{i, j} \gamma_{i, j}(t) \frac{a_{i}^{1} \sigma_{1}^{(i)}(f)^{2}}{a_{i}^{1} \sigma_{1}^{(i)}(f)^{2}+a_{j}^{2} \sigma_{2}^{(j)}(f)^{2}+\sigma^{2}} \mathcal{S} x(t, f), \\
& \widehat{\mathcal{S} s_{2}}(t, f)=\sum_{i, j} \gamma_{i, j}(t) \frac{a_{j}^{2} \sigma_{2}^{(j)}(f)^{2}}{a_{i}^{1} \sigma_{1}^{(i)}(f)^{2}+a_{j}^{2} \sigma_{2}^{(j)}(f)^{2}+\sigma^{2}} \mathcal{S} x(t, f) .
\end{aligned}
$$

Let us consider the orthogonal projection of the estimated sources over the vector space spanned by the real sources. We may write $\hat{s}_{1}=\alpha_{1} s_{1}+\alpha_{2} s_{2}+n_{1}$ and $\hat{s}_{2}=\beta_{1} s_{1}+\beta_{2} s_{2}+n_{2}$.

We define a Source to Interference Ratio (SIR) as the ratio in $\mathrm{dB}$ between the source component $\alpha_{1} s_{1}$ (in the case of the first source $\hat{s}_{1}$ ) and the interference component $\alpha_{2} s_{2}$.

We also define a Source to Artefact Ratio (SAR) as the ratio between the actual mixture $\alpha_{1} s_{1}+\alpha_{2} s_{2}$ and the noise component $n_{1}$. Note that these two components are supposed to be orthogonal

$$
\begin{aligned}
& \operatorname{SIR}_{1}=20 \log _{10}\left|\frac{\alpha_{1}}{\alpha_{2}}\right| \frac{\left\|s_{1}\right\|}{\left\|s_{2}\right\|} \quad \operatorname{SAR}_{1}=20 \log _{10} \frac{\left\|\hat{s}_{1}-n_{1}\right\|}{\left\|n_{1}\right\|} \\
& \mathrm{SIR}_{2}=20 \log _{10}\left|\frac{\beta_{2}}{\beta_{1}}\right| \frac{\left\|s_{2}\right\|}{\left\|s_{1}\right\|} \quad \mathrm{SAR}_{2}=20 \log _{10} \frac{\left\|\hat{s}_{2}-n_{2}\right\|}{\left\|n_{2}\right\|} .
\end{aligned}
$$

The SIR is a way to measure the residual of the other source in the estimation of each source, whereas the SAR is an estimate of the amount of distortion in each estimated signal. One may find more details about these measures in [10].

\section{EXPERIMENTAL STUDY}

In the experimental setting, we work on two tracks of a jazz piece, provided separately on a CD designed to learn how to play jazz. A first track contains the piano and bass part, whereas the second track consists of the drum part. Both tracks are consistent with each other, i.e., when they are mixed, they form a coherent piece of music.

We use 45 s of each excerpt separately as training data, for estimating both source model parameters (PSD vectors and prior weights in the GMM model): one model for the piano + bass track and another model for the drums. This is done using a conventional Expectation-Maximization procedure for optimising the training data likelihood (maximum likelihood criterion).

The next $15 \mathrm{~s}$ of music are mixed by adding both tracks. This excerpt is different from the training excerpts. We estimate the sources in the separation step from the audio mixture, using as prior knowledge the source models estimated in the training phase.

The excerpts are sampled at a sampling rate of $11 \mathrm{kHz}$. As an input to the STFT, we use a windowed signal frame of length $47 \mathrm{~ms}$.

Note that the sources are approximately decorrelated, as $10 \log _{10}\left(\left|\left\langle s_{1}, s_{2}\right\rangle\right| /\left\|s_{1}\right\|\left\|s_{2}\right\|\right)=-16 \mathrm{~dB}$. Indeed, although belonging to the same piece, the sources do not show any short-term correlation, though they obviously are not completely independent.

\section{A. Evaluation}

We evaluate the source to interference ratio (SIR) and the source to artefact ratio (SAR) with various numbers of components $Q_{1}, Q_{2}$ in the mixture models. We evaluate the GMM models and Gaussian scaled mixture models (GSMM). 
TABLE I

SIR FOR EACH OF THE SOURCES AS A FUNCTION OF THE NUMBER OF COMPONENTS IN EACH SOURCE MODEL

\begin{tabular}{c|c|c|c|c|c}
\hline \multicolumn{2}{c|}{ criterion $\rightarrow$} & \multicolumn{2}{c|}{ PM } & \multicolumn{2}{c}{ MAP } \\
\hline \hline state & source & GMM & GSMM & GMM & GSMM \\
\hline \hline Wiener & piano & 8.7 & 4.7 & 15.5 & 4.4 \\
Wiener & drums & 6.7 & 19.6 & 0.7 & 13.8 \\
\hline 4 & piano & 10.5 & 11.0 & 20.0 & 10.5 \\
4 & drums & 9.7 & 18.5 & 2.8 & 18.2 \\
\hline 8 & piano & 11.0 & 11.1 & 20.0 & 10.7 \\
8 & drums & 11.3 & 18.2 & 4.0 & 18.1 \\
\hline 16 & piano & 11.8 & 12.9 & 16.9 & 12.6 \\
16 & drums & 11.9 & 16.9 & 4.2 & 16.3 \\
\hline
\end{tabular}

TABLE II

SAR FOR EACH OF THE SOURCES AS A FUNCTION OF THE NUMBER of COMPONENTS IN EACH SOURCE MODEL

\begin{tabular}{c|c|c|c|c|c}
\hline \multicolumn{2}{c|}{ criterion $\rightarrow$} & \multicolumn{2}{c|}{ PM } & \multicolumn{2}{c}{ MAP } \\
\hline \hline state & source & GMM & GSMM & GMM & GSMM \\
\hline \hline Wiener & piano & 7.8 & 12.3 & 1.8 & 12.4 \\
Wiener & drums & 5.8 & 0.0 & 14.7 & -0.5 \\
\hline 4 & piano & 8.4 & 8.7 & 2.0 & 8.6 \\
4 & drums & 5.9 & 4.0 & 9.6 & 3.7 \\
\hline 8 & piano & 8.9 & 8.5 & 3.4 & 8.7 \\
8 & drums & 5.9 & 4.0 & 8.9 & 4.0 \\
\hline 16 & piano & 8.1 & 8.6 & 3.5 & 8.4 \\
16 & drums & 5.4 & 5.3 & 8.3 & 5.0 \\
\hline
\end{tabular}

The performances are reported in Table I for the SIR and Table II for the SAR. Note that we have also given the SIR and SAR for the standard Wiener filtering, in these tables, as this technique can be seen as a particular case of the proposed method with a single mixture component per model.

\section{B. Discussion}

As the number of Gaussian components in each source model goes from 1 (Wiener standard setting) to four components and then eight components, the SIR and SAR seem to improve. Then with 16 components, the SIR and SAR decrease in some cases (and for some particular estimators) or increase in other cases. This may be interpreted as a consequence of model overfitting, although it might come also from initialization problems in the training step (EM algorithm).

For the GSMM approach with 16 components for each source model, the SIR reaches approximately $12 \mathrm{~dB}$ for the piano + bass source and $16 \mathrm{~dB}$ for the drum source, with an SAR in the range of 9 and $5 \mathrm{~dB}$ respectively. These figures globally represent an improvement compared to the standard Wiener filtering technique, which shows an advantage in using source models that are able to track their statistical behaviors.

We may remark that in the GSMM case, the MAP criterion gives slightly poorer results compared to the PM criterion, although it is computationally less expensive. In the GMM case, the MAP criterion gives poor results.

The GSMM model seems to improve the SIR results compared to the GMM model, in particular for the drum source, at the cost of a slight SAR decrease.

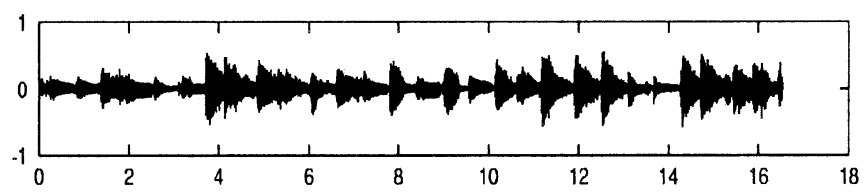

(a)

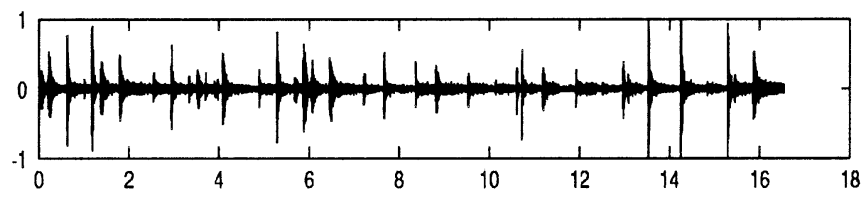

(b)

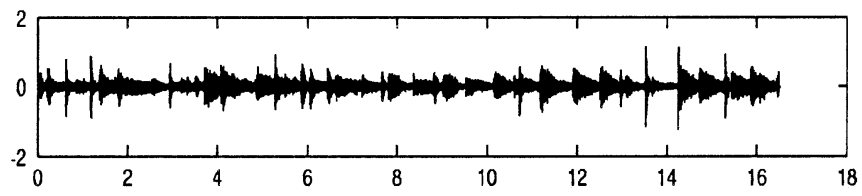

(c)

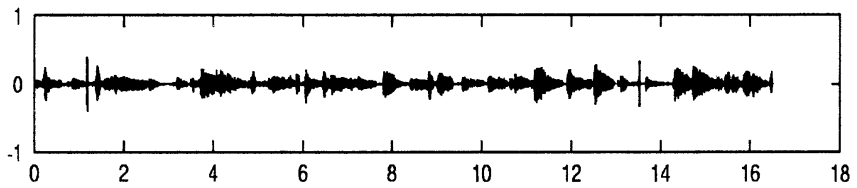

(d)

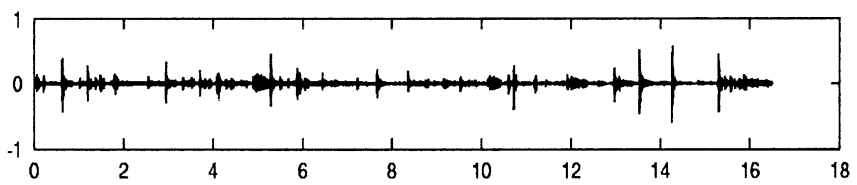

(e)

Fig. 1. (a) Piano + bass source, (b) drum source, (c) mixture of both sources, (d) estimated piano + bass source, and (e) estimated drum source.

In Fig. 1, one can see the waveforms of our excerpt for the original sources, the mixture, and the estimated sources.

It must be underlined that the trends observed in our experiments are undoubtedly dependent on the statistical properties of the two sources used in this study. A more comprehensive experimental investigation, using various sources and different families of models will be necessary before drawing conclusions with a more general significance.

\section{CONCLUSION}

We have presented an approach to single sensor source separation based on an extension of Wiener filtering to nonstationary processes, through the use of Gaussian mixture models instead of plain Gaussian densities in the standard Wiener approach. We have extended the approach to the case of Gaussian scaled mixture models, which permits to advantageously separate the PSD shape from the amplitude information.

The presented approach makes use of a preliminary step, in which PSD vectors are estimated on some excerpts of the sources, corresponding to the various GSMM model states. This prior information is needed in order to perform the source separation. Our preliminary experiments show some benefit on the approach as compared to Wiener filtering, on our example. 
Many tracks deserve to be further investigated to improve and robustify the proposed approach. For instance, the prior densities that we have used in the Bayesian framework are all phase invariant. Thus, we may not recover through these models the true phase of the sources. Phase modeling in the STFT domain should be studied, in order to improve further the approach.

An other step could consist in introducing a psycho-acoustic model (both in the separation step and in the evaluation criteria) in order to optimize the separation in the most perceptible frequency bands for a given source, rather than using a uniform criterion, as is the case in the current approach.

\section{REFERENCES}

[1] S. Amari and J. Cardoso, "Blind source separation-Semiparametric statistical approach," IEEE Trans. Signal Processing, vol. 45, no. 11, pp. 2692-2700, Nov. 1997.

[2] A. J. Bell and T. J. Sejnowski, "An information-maximization approach to blind separation and blind deconvolution," Neural Comput., vol. 7, pp. 1129-1159, 1995

[3] E.-L. Benaroya, "Séparation de plusieurs sources sonores avec un seul microphone," Thèse de Doctorat, Univ. de Rennes I, Rennes, France, Jun. 2003.

[4] L. Benaroya, R. Gribonval, and F. Bimbot, "Non negative sparse representation for wiener based source separation with a single sensor," in Proc. ICASSP, Hong Kong, 2003, pp. 613-616.

[5] O. Bermond and J.-F. Cardoso, "Approximate likelihood for noisy mixtures," in Proc. ICA'99, Aussois, France, 1999, pp. 325-330.

[6] A. Bijaoui, "Wavelets, Gaussian mixtures, and wiener filtering," Signal Process., vol. 82, pp. 709-712, 2002.

[7] J. F. Cardoso, "Blind signal separation: Statistical principles," Proc. IEEE, vol. 86, no. 10, pp. 2009-2025, Oct. 1998.

[8] D. L. Donoho, "Denoising by soft-thresholding," IEEE Trans. Inform. Theory, vol. 41, no. 5, pp. 613-627, May 1995.

[9] Y. Ephraim and N. Merhav, "Hidden Markov processes," IEEE Trans. Inform. Theory, vol. 48, no. 6, pp. 1518-1569, Jun. 2002.

[10] R. Gribonval, L. Benaroya, E. Vincent, and C. Févotte, "Proposals for performance measurement in source separation," in Proc. ICA, Nara, Japan, 2003, pp. 715-720.

[11] A. Hyvärinen and E. Oja, "Independent component analysis: Algorithms and applications," Neural Networks, vol. 13, pp. 411-430, 2000.

[12] D. D. Lee and H. S. Seung, "Algorithms for nonnegative matrix factorization," in Proc. NIPS, 2000, pp. 556-562.

[13] T. Lee, M. Lewicki, M. Girolami, and T. Sejnowski, "Blind source separation of more sources than mixtures using overcomplete representations," IEEE Signal Processing Lett., vol. 6, no. 4, pp. 87-90, Apr. 1999.

[14] J. Portilla, V. Strela, M. J. Wainwright, and E. Simoncelli, "Adaptive wiener denoising using a Gaussian scale of mixture model in the wavelet domain," in Proc. 8th Int. Conf. Image Processing, Thessaloniki, Greece, Oct. 2001.

[15] S. T. Roweis, "One microphone source separation," in Proc. NIPS, 2000, pp. 793-799.

[16] M. Tipping and C. Bishop, "Probabilistic principal component analysis," J. R. Statist. Soc., ser. B, vol. 61, no. 3, pp. 611-622, 1999.

[17] A. P. Varga and R. K. Moore, "Hidden markov model decomposition of speech and noise," in Proc. ICASSP'90, 1990, pp. 845-848.

[18] S. V. Vaseghi and B. P. Milner, "Noise compensation methods for hidden markov model speech recognition in adverse environments," IEEE Trans. Speech Audio Processing, vol. 5, no. 1, pp. 11-21, Jan. 1997.
[19] Y. Zhao, "Frequency-domain maximum likelihood estimation for automatic speech recognition in additive and convolutive noises," IEEE Trans. Speech Audio Processing, vol. 8, no. 3, pp. 255-266, May 2000.

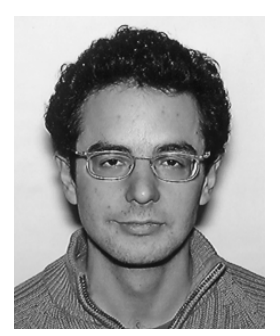

Laurent Benaroya received the M.S. degree from Ecole Centrale Paris (ECP), Paris, France, in 1997 and the Ph.D. degree from IRISA, University of Rennes 1, France, in 2003.

He joined Mist Technologies at the end of year 2003 and is now Research Director. His research interests include Bayesian methods, audio, and speech processing, sparse signal representation.

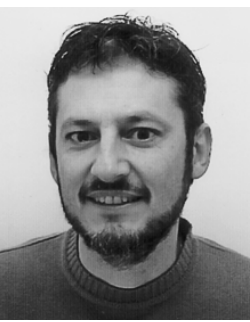

Frédéric Bimbot received the B.A. in degree in linguistics in 1987 from Sorbonne Nouvelle University, Paris III, the telecommunication engineer degree in 1985 from ENST, Paris, France, and the Ph.D. degree in signal processing in 1988.

In 1990, he joined CNRS (French National Center for Scientific Research) as a Permanent Researcher; he was with ENST for 7 years and then moved to IRISA (CNRS \& INRIA), Rennes. He also repeatedly visited AT\&T-Bell Laboratories between 1990 and 1999. He has been involved in several European projects: SPRINT (speech recognition using neural networks), SAM-A (assessment methodology), and DiVAN (audio indexing). He has also been the work-package manager of research activities on speaker verification, in the projects CAVE, PICASSO, and BANCA. His research is focused on audio signal analysis, speech modeling, speaker characterization and verification, speech system assessment methodology, and audio source separation. He is heading the METISS research group at IRISA, dedicated to selected topics in speech and audio processing.

From 1996 to 2000, he was Chairman of the "Groupe Francophone de la Communication Parlée" (now AFCP), and from 1998 to 2003, a member of the ISCA Board (International Speech Communication Association, formerly known as ESCA).

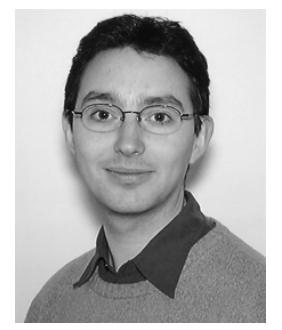

Rémi Gribonval graduated from Ecole Normale Supérieure, Paris, France in 1997. He received the Ph.D. degree in applied mathematics from the University of Paris-IX Dauphine, Paris, France, in 1999.

From 1999 to 2001, he was a visiting scholar at the Industrial Mathematics Institute (IMI) in the Department of Mathematics, University of South Carolina. $\mathrm{He}$ is currently a Research Associate with the French National Center for Computer Science and Control (INRIA) at IRISA, Rennes, France. His research interests are in adaptive techniques for the representation and classification of audio signals with redundant systems, with a particular emphasis in blind audio source separation. 